\title{
DFT Calculations: A Powerful Tool for Materials Design
}

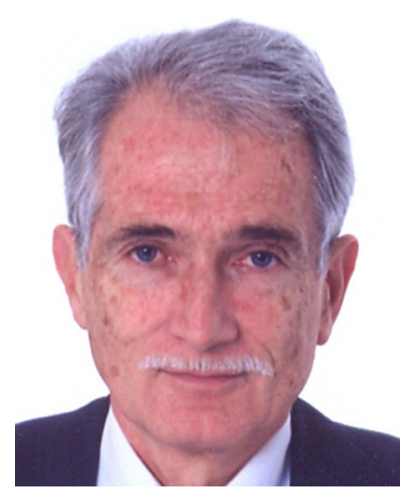

Peter Rogl

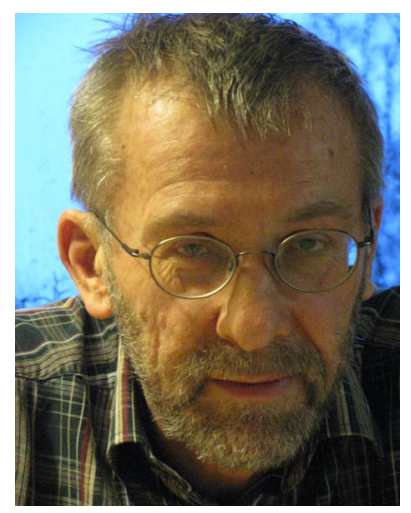

Raimund Podloucky

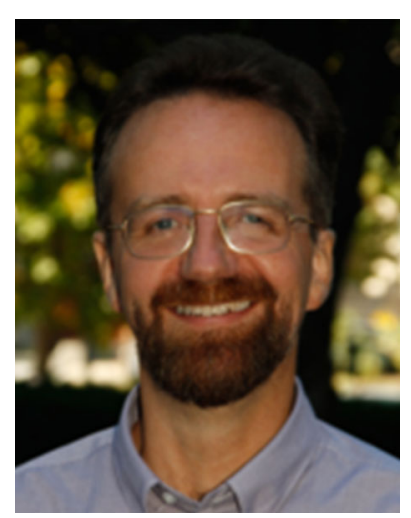

Walter Wolf
Most of us are using advanced computational approaches based on density functional theory (DFT) and other methods that are able to predict materials properties. First principle calculations conduct accurate analyses and are better and better able to calculate materials properties at atomic/electronic levels based on quantum mechanics, statistical thermodynamics, classical mechanics and electrodynamics. Although first principles calculations require an enormous amount of calculations, they nowadays are able to provide rapid and reliable answers for a range of materials issues related to materials design for electrical power generation, automotive applications, energy storage, microelectronics and serving the chemical industry in general. The recent advancement of computer power-increasing by about 1000 times over the past ten years - has provided a strong driving force for computational science. For comparison the Japanese K-computer performed 10.5 PFLOPS in 2011 (the world's fastest computer equivalent to $10^{16}$ operations per second). In addition, significant computational power of a couple hundred processors can nowadays be assembled in a desktop-like device, which makes high-end computational simulation available for essentially everyone. Under the innovative High Performance Computing Infrastructure connecting the K-supercomputer and major supercomputers at universities and research institutes, Japan has made efforts to run computation programs more efficiently, developing first principles molecular dynamics calculation programs such as PHASE or CONQUEST. Similarly the National Institute of Standards and Technology (NIST) announced activities to establish a new NIST-sponsored centre of excellence for advanced materials research: Center for Hierarchical Materials Development (CHiMaD). It is said that the new centre will focus on the development of the next generation of computing tools, databases as well as experimental techniques for a knowledge driven design of materials.

In general, the dramatically increased leverage of quantum physical simulations in all areas of materials research is not only triggered by the rapidly increased availability of computational power, but also by tremendous progress made in the development of versatile and robust computational algorithms. First-principles methods are based on the solution of Schrödinger's equation describing the quantised interaction between a large number of charged particles, i.e. electrons and nuclei. This breath-takingly complex problem is recast by DFT in terms of orbital-like equations, in which all quantum physical interactions are packed into the so-called exchange correlation potential, for which no feasible expression can be made for a realistic system of interacting atoms. Therefore, approximations have to be made and the quality of the results depends on the quality of these approximations. It turned out that for many systems (i.e. for which the electronic states are not strongly localised) the results are reliable and useful. In other cases, such as transition metal oxides, rare-earth compounds, van der Waals interactions etc. the approximations can be improved by so-called post DFT approaches, which are computationally more demanding but still feasible. By resolving these quantum physics complications, first-principles calculations remain free from any empirical information and its predictive power is of particular interest searching for new materials with hitherto unknown properties. On the other hand, basic thermodynamical properties such as heat of formation can be calculated at least as reliable as obtained from experiment but much faster and for many more possible structures and compositions, which helps to find the true thermodynamical ground state. Based on DFT electronic structure calculations also forces on atoms and stresses can be easily derived, which is needed for optimizing structural parameters and for deriving vibrational properties. Including phonons enormously widens the range of applications for DFT studies because most of the temperature effects of the free energy can be included. DFT calculations should not only be considered to be useful for studying some selected cases, i.e. compounds with a given composition, but they are very useful to produce a data basis for searching a large space of possible structural configurations. This can be done by making use of the so-called Cluster Expansion for which the effective interactions between 
structural elements on a lattice are derived from a reasonably large set of first-principles calculations. When including the vibrational free energy (derived by the same first-principles procedure) into the effective interaction energies phase diagrams and microstructural evolution can be explored on the computer screen purely based on the fundamental quantum physical equations as given by DFT, free from any empirical parameters. Alternatively, ab initio molecular dynamics trajectories can be used to fit the adjustable parameters of so-called force fields, such as embedded atom (EAM) or charge optimized many body (COMB) potentials, which then allow classical molecular dynamics simulations for much larger lengths and time scales, e.g. for modelling the evolution of dislocations, loops or phase boundaries. Cluster expansion and force field fitting allow extending the accuracy and predictive power of first principles methods to much larger ensembles and time frames, far beyond feasibility for the DFT methods alone.

Rapid increase of computational power and the development of sophisticated and reliable algorithms are prerequisites of the current success of fist principles based simulation in materials science. Yet another indispensable prerequisite for an increasingly widespread application of these methods is the availability of computational modelling and simulation environments, such as Materials Design's MedeA platform, which help expert as well as non-expert users, experimentalists and engineers, to achieve their research goals by complementing their expertise with easily accessible high-end simulations. As a tremendous productivity tool such environments enable easy model building, access to structure databases, fast set-up of simulations from graphical user interfaces, versatile combination of computational methods such as discussed above by graphical flow chart building, and fully automatic execution of complex simulation protocols on in-house or external compute facilities without any further user interaction. In addition, access to computational results and their analysis is made easy, such that the overall demand in man-power for setup, running and analysing such complex simulations is dramatically decreased. Such modelling environments are critically important to facilitate access to efficient and highly reliable computational modelling both for simulation experts as well as researchers and engineers with expertise in many other areas of materials science and engineering.

The enormously wide scope of the current computational science can be best seen from the Journal "Computational Materials Science", which "invites contributions on quantum, classical and statistical mechanical studies, such as, but not limited to: semiempirical, e.g. tight-binding and embedded-atom methods; first-principle calculations; density-functional theory; atomic and molecular-scale simulations, e.g. MC and MD techniques; other modelling techniques using macroscopic input, e.g. FEmethods, and contributions on properties of materials, including electronic, dynamical, transport, mechanical, growth and thermodynamical properties of materials such as metals and alloys, semiconductors, insulators, superconductors, biomaterials, polymers, ceramics and composites in liquid, crystal, amorphous and cluster-like states".

The future will likely form a close link between experimental and ab-initio techniques, which particularly with experimentally inaccessible data provide valuable support in materials design.

\author{
Peter Rogl \\ Associate Editor \\ Journal of Phase Equilibria and Diffusion \\ University of Vienna \\ Institute of Physical Chemistry \\ Währingerstrasse 42 A-1090 Vienna, Austria \\ E-mail: peter.franz.rogl@univie.ac.at
}

Raimund Podloucky

University of Vienna

Institute of Physical Chemistry

Sensengasse 8 / 7 A-1090 Vienna, Austria

E-mail: Raimund.Podloucky@univie.ac.at

\author{
Walter Wolf \\ Materials Design s.a.r.l. \\ 18, rue de Saisset 92120 Montrouge, France \\ E-mail: wwolf@materialsdesign.com
}

